\title{
PERFIL BIOCLIMÁTICO PARA CRIAÇÃO DE VACAS LEITEIRAS NA REGIÃO DE SANTARÉM, PARÁ
}

\author{
Jamille Debora de oliveira Batista ${ }^{1}$; Sullyvan Silva Oliveira²; Monique Almeidaa ${ }^{3}$; Nara de Medeiros \\ Pordeus4; Nayara Lima Pereira5; Alanna do Socorro Lima da Silva6; Graciene Conceição dos Santos ${ }^{7}$. \\ ${ }^{1}$ Universidade Federal da Grande Dourados, Dourados, Mato Grosso do Sul, Brasil, jamilledeboraob@gmail.com \\ 2 Universidade Federal do Oeste do Pará, Santarém, Pará, Brasil, sullyvanoliveira23@gmail.com \\ 3 Universidade Federal do Oeste do Pará, Santarém, Pará, Brasil, moniquealmeida317@gmail.com \\ ${ }^{4}$ Universidade Federal do Oeste do Pará, Santarém, Pará, Brasil, pordeusn@gmail.com \\ ${ }^{5}$ Universidade Federal do Oeste do Pará, Santarém, Pará, Brasil, naylima.pereira@gmail.com \\ ${ }^{6}$ Universidade Federal do Oeste do Pará, Santarém, Pará, Brasil, aslsilva@yahoo.com.br \\ 7 Universidade Federal do Oeste do Pará, Santarém, Pará, Brasil, gracienecsantos@yahoo.com.br.
}

RESUMO: O objetivo do presente trabalho foi avaliar as condições climáticas e fazer um diagnóstico bioclimático para a produção leiteira na região de Santarém - PA. Foram utilizados os dados referentes ao período de doze meses (janeiro a dezembro de 2015). As variáveis climáticas consideradas para a caracterização foram: média da Temperatura máxima $\left(\operatorname{TMAX},{ }^{\circ} \mathrm{C}\right)$ e Temperatura mínima $\left(\mathrm{TMIN},{ }^{\circ} \mathrm{C}\right)$, média da umidade relativa do ar (UR, \%) e índice de temperatura e umidade (ITU) dos doze meses. De acordo com o perfil bioclimatológico traçado da região, é preciso adotar práticas de manejo para auxiliar os animais na manutenção do conforto térmico nas épocas mais críticas (Jul - Dez), minimizando a influência negativa sobre seu sistema fisiológico, consequentemente, evitando a queda na produção e qualidade do leite.

PALAVRAS-CHAVE: Índice de temperatura, Umidade, Variáveis climáticas.

\section{BIOCLIMATIC PROFILE FOR DAIRY CATTLE BREEDING IN THE REGION OF SANTARÉM, PARÁ}

ABSTRACT: The objective of this work was to evaluate the climatic conditions and from this evaluation to perform a bioclimatic diagnosis for milk production in the region of Santarém - PA. The data used refer to a period of twelve months (from January to December 2015). The following were considered as the climatic variables considered for the characterization: mean maximum temperature $\left(\operatorname{TMAX}^{\circ}{ }^{\circ} \mathrm{C}\right)$ and minimum temperature $\left(\mathrm{TMIN}^{\circ}{ }^{\circ} \mathrm{C}\right)$, mean relative humidity $(\mathrm{RH}, \%)$ and temperature and humidity index ) obtained in the twelve months studied. According to the bioclimatological profile of the region, it is necessary to adopt management practices to assist the animals in the maintenance of thermal comfort in the most critical times (Jul - Dec), minimizing the negative influence on their physiological system, consequently avoiding the fall in production and quality of milk. 
KEYWORDS: Temperature, Climate variables, Temperature and humidity index.

\section{PERFIL BIOCLIMÁTICO PARA LA CRÍA DE VACAS LECHERAS EN LA REGIÓN DE SANTARÉM, PARÁ}

RESUMEN: El presente trabajo objetivó evaluar las condiciones climáticas y a partir de esta evaluación realizar un diagnóstico bioclimático para la producción lechera en la región de Santarém - PA. Los datos utilizados se refieren a un período de doce meses (de enero a diciembre de 2015). Se tomaron como las variables climáticas consideradas para la caracterización: promedio de la temperatura máxima (TMAX, C) y Temperatura mínima ( $\left.\mathrm{TMIN}^{\circ}{ }^{\circ} \mathrm{C}\right)$, media de humedad relativa del aire (UR,\%) y índice de temperatura y humedad (ITU ) obtenidos en los doce meses estudiados. De acuerdo con el perfil bioclimatológico trazado de la región, es necesario adoptar prácticas de manejo para auxiliar a los animales en el mantenimiento del confort térmico en las épocas más críticas (Jul - Diez), minimizando la influencia negativa sobre su sistema fisiológico, consecuentemente, evitando la caída en la caída producción y calidad de la leche.

PALABRAS CLAVE: Indice de temperatura, Humedad, Variables climáticas.

O Brasil é tradicionalmente um grande produtor de leite, uma atividade que começou com características extrativistas, e ocupa posição de destaque no cenário econômico nacional, sendo, atualmente, um dos principais agronegócios do Brasil (SIQUEIRA et al., 2010). Conforme dados do IBGE, em 2015, foram captados pela indústria nacional cerca de 35 bilhões de litros de leite e a produtividade média foi de
1.609 litros/vaca/ano. Entre os principais estados na captação de leite, Minas Gerais continuou com o maior volume de leite captado (6,4 bilhões de litros), seguido pelo Rio Grande do Sul (3,5 bilhões de litros), Paraná (2,8 bilhões de litros), São Paulo (2,6 bilhões de litros), Goiás (2,4 bilhões de litros) e Santa Catarina (2,3 bilhões de litros) (IBGE, 2016).

Para a região Norte os dados apontam para uma expressiva taxa de 
crescimento dentro desta atividade. No período de 1990 a 2001, essa taxa foi de $41,6 \%$ para o Brasil como um todo, pouco mais de $10 \%$ para a região nordeste, quase $24 \%$ para o sudeste, $59 \%$ para a região sul e $91 \%$ para o centro-oeste, no entanto, foi de 122,7\% para a região norte (BRESSAN; VILELA, 2003). Em 2015, a quantidade de leite adquirido na região norte foi de aproximadamente 1 bilhão de litros, tendo o Pará registrado o volume de 234.603 mil litros de leite adquirido, colocando-o como o segundo maior produtor do estado (IBGE, 2016).

Ainda assim, esses valores comprovam a baixa produtividade brasileira, havendo a necessidade de melhorar a eficiência com que o leite é produzido. Nesse aspecto, a própria condição climática do país acaba interferindo nas características quantitativas e qualitativas do leite. Sabe-se que dois terços do território brasileiro está situado em região tropical, onde há predominância de temperaturas elevadas (AZEVEDO et al., 2005). Essa condiç̧ão piora os efeitos do estresse térmico e limita o desenvolvimento, a produção e a reprodução dos animais (AVENDANO et al., 2006). Em ambientes tropicais a magnitude do fator térmico é causada pelo efeito combinado de alta temperatura e umidade relativa do ar, elevada incidência de radiação solar e baixa velocidade do vento, o que reduz a eficiência da perda de calor (DIKMEN; HANSEN, 2009)

De todo modo a temperatura ótima para exploração leiteira depende de diversos fatores, como espécie, raça, idade, consumo alimentar, aclimatação, nível de produção, pelame e grau de tolerância do animal ao calor e ao frio (ALMEIDA NETO et al., 2011) e para se obter um melhor desempenho do animal é necessário que se dê importância ao clima, em que estes se encontram, tendo em vista que os elementos climáticos influenciam diretamente na produção (SOUZA, et al. 2010). 
Assim, com base nesse contexto,

Foram utilizados os dados objetivou-se avaliar as condições referentes ao período de doze meses climáticas e traçar um perfil (janeiro a dezembro de 2015). As bioclimático para o desenvolvimento variáveis climáticas consideradas para a da atividade leiteira na região de caracterização foram: média da Santarém - PA, e assim auxiliar no entendimento dos efeitos do clima sobre a produção.

A caracterização do bioclima da região de Santarém $\left(02^{\circ} 26^{\prime} 18^{\prime \prime} S\right.$ e

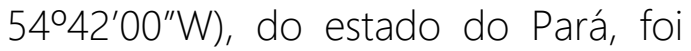
realizada com os dados climáticos do Temperatura máxima $\left(\operatorname{TMAX},{ }^{\circ} \mathrm{C}\right)$ e Temperatura mínima $\left(\mathrm{TMIN},{ }^{\circ} \mathrm{C}\right)$, média da umidade relativa do ar (UR, \%) e índice de temperatura e umidade (ITU) dos doze meses, calculado com base na equação proposta por Thom (1959) com base nas médias da temperatura Instituto Nacional de Meteorologia e umidade relativa: (INMET).

em que:

$$
I T U=t_{m}+0,36 t_{p o}+41,5
$$

$t_{m}$ - temperatura média do $\mathrm{ar}^{\circ}{ }^{\circ} \mathrm{C}$;

$t_{p o}$ - temperatura do ponto de orvalho, expressa em ${ }^{\circ} \mathrm{C}$, calculada a partir da combinação das equações psicrométricas citadas por Vianello e Alves (2000):

em que:

$$
t_{p o}=\frac{186,4905-237,3 \log _{10}(\text { UR es } / 100)}{\log _{10}(\text { UR es } / 100)-8,2859}
$$

$U R$ - umidade relativa do ar, \%;

es - pressão de saturação de vapor d'água no ar, $\mathrm{hPa}$, estimada pela expressão:es = $6,107810\left(\frac{7,5 t}{237,3+t}\right)$

Os dados coletados sobre o diagnóstico bioclimático de Santarém e que serviram de base para o presente estudo estão apresentados na Tabela 1. Os dados climatológicos foram comparados com as condições de conforto térmico ideais para vacas leiteiras, com base nas recomendações de temperatura citadas por Pereira (2005) que estabelece para bovinos mestiços zona termoneutra entre $5 \mathrm{e}$ $31^{\circ} \mathrm{C}$, visto que a grande parte dos 
animais produtores de leite da região são animais cruzados.

Com relação à umidade relativa, adotou-se a recomendação de Sampaio et al. (2004) com valores entre 55 e 70\% como característica desejável para facilitar os processos evaporativos para a perda de calor.
Os valores de ITU foram confrontados com as recomendações de Lima et al. (2007), que considera para animais de produção os valores de ITU iguais ou menores que 75 caracterizam situação normal, em alerta de 75 a 78 , perigo de 79 a 83 e de emergência quando for maior que ou igual a 84 .

Tabela 1. Médias das temperaturas máximas e mínimas, umidade relativa do ar, índice de temperatura e umidade incidente em Santarém, Pará.

\begin{tabular}{lcccc}
\hline Meses & $\begin{array}{c}\text { Temperatura } \\
\text { máxima } \\
\left({ }^{\circ} \mathrm{C}\right)\end{array}$ & $\begin{array}{c}\text { Temperatura } \\
\text { mínima } \\
\left({ }^{\circ} \mathrm{C}\right)\end{array}$ & $\begin{array}{c}\text { Umidade } \\
\text { Relativa } \\
(\%)\end{array}$ & $\begin{array}{c}\text { índice de temperatura e } \\
\text { umidade }\end{array}$ \\
\hline Janeiro & 30,94 & 22,35 & 86,86 & 77,24 \\
Fevereiro & 30,05 & 22,11 & 89,25 & 76,93 \\
Março & 30,00 & 21,80 & 91,42 & 76,75 \\
Abril & 30,75 & 22,17 & 88,84 & 77,57 \\
Maio & 30,46 & 22,28 & 90,51 & 77,53 \\
Junho & 30,93 & 22,18 & 89,37 & 77,84 \\
Julho & 31,25 & 22,09 & 88,44 & 77,81 \\
Agosto & 32,84 & 22,46 & 83,90 & 78,99 \\
Setembro & 33,77 & 23,02 & 80,70 & 79,39 \\
& 34,22 & 23,36 & 78,75 & 79,76 \\
Outubro & 34,55 & 23,20 & 79,40 & 80,19 \\
Novembro & 34,09 & 23,42 & 79,48 & 79,81 \\
Dezembro & & & & \\
\hline
\end{tabular}

Fonte: INMET (2015).

Na Tabela 2 foram minúscula sublinhada a UR e a quarta apresentados $\quad$ resultado da letra maiúscula sublinhada ao ITU. Para comparação das exigências térmicas efeito de avaliação considerou-se a das vacas leiteiras na região de simbologia para a avaliação da Santarém. A primeira letra maiúscula temperatura como sendo: $(I)$ = inferior, refere-se à TMAX; a segunda letra (C) = confortável e (S) = superior aos minúscula a TMIN; a terceira letra valores climáticos exigidos. 
Tabela 2. Diagnóstico bioclimático para produção de vacas leiteiras na região de Santarém, Pará.

\begin{tabular}{|c|c|}
\hline Meses & Diagnóstico para vacas leiteiras \\
\hline Janeiro & $\operatorname{CcsS}$ \\
\hline Fevereiro & $\operatorname{CcsS}$ \\
\hline Março & $\operatorname{Ccs} \underline{\bar{S}}$ \\
\hline Abril & $\operatorname{CcsS}$ \\
\hline Maio & $\operatorname{CcsS}$ \\
\hline Junho & $\operatorname{Ccs}$ \\
\hline Julho & $\operatorname{Scs} \underline{S}$ \\
\hline Agosto & $\operatorname{scsS}$ \\
\hline Setembro & $\operatorname{ScsS}$ \\
\hline Outubro & ScsS \\
\hline Novembro & ScsS \\
\hline Dezembro & ScsS \\
\hline
\end{tabular}

Primeira letra maiúscula: TMAX; segunda letra minúscula: TMIN; terceira letra minúscula sublinhada: UR; quarta letra maiúscula sublinhada: ITU. Para efeito de avaliação considerou-se: $(I)=$ inferior, $(C)=$ confortável e $(S)$ = superior aos valores climáticos exigidos.

É possível observar na Tabela 2 temperatura corporal e na frequência que no período de julho a dezembro foram registradas temperaturas respiratória (WEST, 2003). Em ambiente máximas superiores ao ideal com temperaturas acima da zona preconizado para gado leiteiro no município de Santarém, com valor máximo de $33,78^{\circ} \mathrm{C}$ em novembro, entretanto, a temperatura mínima se manteve na zona de conforto durante os doze meses.

Animais submetidos a ambientes com temperaturas superiores aos níveis de conforto térmico, respondem ao estresse por calor com redução no consumo de alimentos e na produção de leite e com aumento na termoneutra ocorre uma diminuição na produção de leite porque os animais reduzem a ingestão de matéria seca (NRC, 2001), indicando aos produtores que devem modificar o ambiente, visto que a UR nesta fase está acima do índice considerado confortável. Modificações primárias como provimento de sombra por árvores ou com uso de telas para minimizar as perdas decorrentes do estresse calórico (BARBOSA et al., 2004; CRUZ et al., 2011), ou construções zootécnicas 
que possuam equipamentos de refrigeração e ventilação projetados especialmente para reduzir a temperatura do ar em abrigos para animais podem ser bastante eficientes (BRASIL; SILVA, 2010).

Com relação aos valores médios de umidade relativa (UR), estes apresentam-se elevados durante todo o ano quando comparado aos valores recomendados por Sampaio et al. (2004) que são entre 55 e 70\%, sendo o mais alto valor observado neste trabalho no mês de fevereiro $(92,74 \%)$ e o mais baixo no mês de dezembro (78,81\%). Malheiros Filho et al. (2009) observaram o maior valor de UR no mês de junho (92\%) e o mais baixo no mês de dezembro (74\%) na região do Brejo Paraibano. Grassmann et al. (2011) encontraram em um experimento com vacas holandesas em Santa Catarina valores da umidade relativa do ar para o período experimental (Setembro de 2010 Junho de 2011) de 73,75\% a 91,41\%,

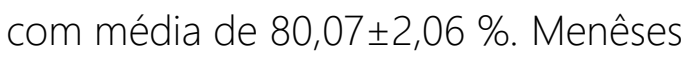

et al. (2016) obtiveram médias variando de 65 a 85\% e definiram a UR como a variável mais instável ao longo do ano, sendo assim o fator que mais interfere no ITU. Esta condição ambiental de elevada umidade relativa do ar é prejudicial ao animal no tocante à perda de calor para o ambiente, especificamente nos mecanismos não evaporativos (condução, convecção e irradiação) que se tornam ineficientes (SILVA, 2000; PEREIRA, 2005), sendo que nessas condições, as vacas reduzem voluntariamente o consumo de alimentos volumosos e, como consequência, o teor de gordura do leite diminui e distúrbios digestivos aumentam (AZEVEDO; ALVES, 2009).

A distribuição de frequência dos valores de ITU para os meses de janeiro a dezembro encontra-se acima da faixa de bem estar animal (Tabela 2) que é de 75, conforme indicações de Lima et al. (2007). Entre outubro e novembro, o ITU apresentou valores maiores que 79 configurando situação de perigo para conforto térmico dos animais. 
Collier et al. (2009) e Collier et al. (2012) afirmaram que reflexos negativos já podem ser observados em ITU acima de 68. Em geral, vacas holandesas em lactação apresentam declínio na produção de leite a partir de ITU igual a 72 (JOHNSON,1987). Para Machado (1998), o valor de ITU igual a 72 pode ser alcançado com temperaturas do ar próximas de $23^{\circ} \mathrm{C}$, e quando a umidade relativa do ar for superior a 95\%, ou quando a temperatura do ar for de $25^{\circ} \mathrm{C}$ e com umidade de $50 \%$.

Como consequências dos elevados valores do índice de temperatura e umidade (ITU) tem sido relatados problemas tanto na produção de leite (KLOSOWSKI et al., 2002; LEBLANC, 2010; RODRIGUES et al, 2010) quanto na atividade reprodutiva dos animais (DE RENSIS; SCARAMUZZI, 2003; COSTA-E-SILVA et al., 2009; FLAMENBAUM; GALON, 2010;). Notase que quando o ITU no ambiente é reduzido, promove uma condição considerada não crítica ao conforto térmico animal, resultando na minimização das perdas na produção de leite (SILVA et al., 2002). Uma das estratégias adotadas para aumentar tanto a produção, como a adaptação dos animais aos trópicos é a utilização do cruzamento de raças especializadas originárias de clima temperado com animais adaptados de raças zebuínas (AZEVEDO; ALVES, 2009; FAÇANHA et al., 2013).

O perfil bioclimatológico traçado da região de Santarém, sinalizou que é preciso adotar práticas de manejo para auxiliar na manutenção do conforto térmico dos animais nas épocas mais críticas (Jul - Dez), minimizando a influência negativa sobre seu sistema fisiológico e, consequentemente, evitando a queda na produção e qualidade do leite.

\section{REFERÊNCIAS}

ALMEIDA NETO, L. A.; PANDORFI, H.; ALMEIDA, G. L. P.; GUISELINI, C. Uso do sistema de resfriamento adiabático evaporativo no conforto térmico de vacas da raça girolando, Revista Brasileira de Engenharia Agrícola e Ambiental, v.15, n.7, p.754-760, Campina Grande, 2011. 
AVENDANO, R. L.; ALVAREZ, V. F. D.; CORREA, C. A.; SAUCEDO, Q. J. S.; ROBINSON, P. H.; FADEL, J. G. Effect of cooling Holstein cows during the dry period on posíparíum performance under heat stress conditions. Livestock Production Science, v.105, n.1-3, p.198206, 2006.

AZEVEDO, D. M. M. R.; ALVES, A. A.; FEITOSA, F. S.; et al. Adaptabilidade de bovinos da raça Pé-Duro às condições climáticas do semiárido do estado do Piauí. Archivos de Zootecnia, v.57, p.513-523, 2008.

AZEVEDO, M.; PIRES, M. F. A.; SATURNINO, H. M.; LANA, A. M. Q.; SAMPAIO, I. B. M.; MONTEIRO, J. B. N.; MORATO, L. E. Estimativa de Níveis Críticos Superiores do Índice de Temperatura e Umidade para Vacas Leiteiras 1/2, 3/4 e 7/8 Holandês-Zebu em Lactação. Revista Brasileira de Zootecnia, Viçosa, v. 34, n. 6, p. $2000-$ 2008, 2005.

BARBOSA, O.R.; BOZA, P.R.; SANTOS, G.T.; SAKAGUSHI, E.S.; RIBAS, N.P. Efeitos da sombra e da aspersão de água na produção de leite de vacas da raça Holandesa durante o verão. Acta Scientiarum. Animal Sciences, v.26, n.1, p.115-122, 2004.

BRASIL, R. B.; SILVA. M. A. P. Conforto térmico na bovinocultura de leite: Revisão bibliográfica. PUBVET, Londrina, v.4, n.15, ed.120, art.813, 2010.

BRESSAN, M.; VILELA, D. Levantamento ex ante de restrições ao desenvolvimento da cadeia produtiva do leite da região Norte. In: Workshop sobre identificação das principais restrições ao desenvolvimento da cadeia produtiva do leite da região norte do brasil, 1, 2003, Juiz de Fora. Anais... Juiz de Fora: Embrapa Gado de Leite, 2003. p. 47-52.

COLLIER, R. J.; HALL, L. W.; RUNGRUANG, S.; ZIMBLEMAN, R. B. Quantifying heat stress and its impact on metabolism and performance. Department of Animal Sciences, University of Arizona, 2012.

COLLIER, R. J.; ZIMBELMAN, R. B.; RHOADS, R.P.; RHOADS, M. L.; BAUMGARD, L. H. A re-evaluation of the impact of temperature humidity index (THI) and black globe humidity index $(\mathrm{BGHI})$ on milk production in high producing dairy cows. Proceedings of the Southwest Nutrition and Management Conference, Arizona, 2009. p. 26-27.

COSTA-E-SILVA, E. V.; RUEDA, P. M.; MONTANARI, J.; RANGEL, R.; ZÚCCARI, C. E. S. N. Bem-estar, ambiência e saúde animal, Ciência animal brasileira, v. 0, n. 0, 2009. Disponível em: < http://revistas.bvs-

vet.org.br/cab/article/view/24450>. Acesso em: 25 nov. 2016.

CRUZ, L.V.; ANGRIMANI, D.S.R.; RUI, B.R. SILVA, M.A. Efeitos do estresse térmico na produção leiteira: revisão de literatura. Revista Científica Eletrônica de Medicina Veterinária, n.16, 2011. 
DE RENSIS, F.; SCARAMUZZI, J. R. Heat Stress and seasonal effects on reproduction in the dairy cow - a review. Theriogenology, v.60, n. 6, p. 1139-1151, 2003.

DIKMEN, S.; HANSEN, P. J. Is the temperature-humidity index the best indicator of heat stress in lactating dairy cows in a subtropical environment? Journal of Dairy Science, v. 92, n. 1, p. 109-116, 2009.

FAÇANHA, D. A. E.; SILVA, R. G.; MAIA, A. S. C. GUILHERMINO, M. M. VASCONCELOS, A. M. Variação anual de características morfológicas e da temperatura de superfície do pelame de vacas da raça Holandesa em ambiente semiárido. Revista Brasileira de Zootecnia, v. 39, n. 4, p. 837-844, 2010.

FLAMENBAUM, I.; GALON, N. Management of heat stress to improve fertility in dairy cows in Israel. Journal of Reproduction and Development, v. 56, p. S36-S41, 2010.

GRASSMANN, C.; EISING, R.; NEVES, L. O.; ELI, K.; JUFFO, E. E. L. Avaliação do índice de temperatura e umidade para vacas leiteiras da raça holandesa em Rio Do Sul, SC, 2011. Disponível em <http://eventos.ifc.edu.br/wpcontent/uploads/sites/5/2014/09/CAZ38.pdf>. Acesso em: 25 nov. 2016.

JOHNSON, K. G. Shading behaviour of sheep: preliminary studies of its relation to thermoregulation, feed and water intakes, and metabolic rates. Australian
Journal Research, v. 38, n. 4, p.587-596, 1987.

KLOSOWSKI, E. S.; CAMPOS, A. T.; CAMPOS, A. C. de; GASPARINO, E. Estimativa no declínio na produção de leite, em período de verão, para Maringá, PR. Revista Brasileira de Agrometeorologia, v. 10, n. 2, p. 283288, 2002.

LEBLANC, S. Assessing the association of the level of milk production with reproductive performance in dairy catle. Journal of Reproduction and Development, v. 56, Sulp, 1-7, 2010.

LIMA, K.A.O.; MOURA, D.J.; NAAS, I.A.; PERISSINOTTO. Estudo da influência das ondas de calor sobre a produção de leite no Estado de São Paulo. Revista Brasileira de Engenharia de Biossistemas, v.1, n.1, p.70-81, 2007.

MACHADO, P. F. Efeitos da alta temperatura sobre a produção, reprodução e sanidade de bovinos leiteiros. In: SIMPÓSIO BRASILEIRO DE AMBIÊNCIA NA PRODUÇÃO DE LEITE. Anais..., 1998.Piracicaba. Anais... Piracicaba: FEALQ, 1998. p. 179-188.

MALHEIROS FILHO, J. R.; CAMERINI, N. L.; SOARES, E. A.; NASCIMENTO, J. W. B.; FURTADO, D. A.; DANTAS, C. P. Estudo bioclimático para gado leiteiro em Município do Brejo Paraibano. Educação Agrícola Superior, v.24, n.1, p.20-22, 2009.

MENÊSES, H. J. S.; ALVES, A. R.; SILVA, A. C.; SILVA, T. A.; SANTOS, F. M. G.; 
SANTOS, A. S. S. Diagnóstico bioclimático para animais ruminantes na região dos Cocais - MA. Anais...X Simpósio Paraibano de Zootecnia, Areia, 2016.

NRC, Nutrient requirements of domestic animals: Nutrient requirements of dairy cattle. $7^{\circ}$ ed.; Washington DC: 2001.

PEREIRA, C. C. J. Fundamentos de Bioclimatologia Aplicados à Produção Animal. Belo Horizonte: FEPMVZ, 2005.

RODRIGUES, A. L.; SOUZA, B. B.; PEREIRA FILHO, $M$. Influência do sombreamento e dos sistemas de resfriamento no conforto térmico de vacas leiteiras, Agropecuária Científica no Semi-Árido, v. 6, n. 2, p. 14-22, 2010.

SAMPAIO, C. A. P.; CRISTANI, J.; DUBIELA, J.A.; BOFF, C. E.; OLIVEIRA, M. A.. Avaliação do ambiente térmico em instalações para crescimento e terminação de suínos utilizando os índices de conforto térmico nas condições tropicais. Revista Ciência Rural, v. 34, n. 3, p. 785-790, 2004.

SILVA, R. G. Introdução à bioclimatologia animal. São Paulo: Nobel, 2000, 286p.

SILVA, I.J.O.; PANDORF, H.; ACARARO Jr., I.; PIEDADE, S. M. S.; MOURA, D. J. Efeitos da climatização do curral de espera na produção de leite de vacas holandesas. Revista Brasileira de Zootecnia, v. 31, n. 5, p.2036-2042, 2002.
SIQUEIRA, K. B.; CARNEIRO, A. V.; ALMEIDA, M. F.; NALON, R. C. S. O mercado lácteo brasileiro no contexto mundial. Circular Técnica 104. Juiz de Fora, Embrapa - Centro Nacional de Pesquisa de Gado de Leite, 2010.

SOUZA, B.B.; SILVA, I. J. O.; MELLACE, E. M.; SANTOS, R. F. S.; ZOTTI, C. A.; GARCIA, P. R. Avaliação do ambiente físico promovido pelo sombreamento sobre o processo termorregulatório em novilhas leiteiras. Agropecuária Científica no Semiárido, v. 06, n. 02, p. 59 - 65, 2010.

THOM, E. C. The discomfort index. Weatherwise, v.12, p.57-60, 1959.

VIANELLO, R. L.; ALVES, A. R. Meteorologia básica e aplicações. Viçosa: UFV, 2000. 448p.

WEST, J.W. Effects of heat-stress on roduction in dairy cattle. Journal of Dairy Science, v.86, n.6, p.2131-2144, 2003. 